\title{
Galactic Center ADAF Ruled out By Polarization
}

\author{
Eric Agol \\ Physics and Astronomy, Johns Hopkins University, Baltimore, MD, 21218 \\ Currently at: Theoretical Astrophysics, Caltech MS 130-33, Pasadena, CA 91125
}

\begin{abstract}
Recent observations of linear polarization at high frequency towards the galactic center radio source, Sgr A*, place strong constraints on the nature of the radio emission region. We discuss how these constraints rule out the larger accretion rate need for low efficiency accretion flows, such as advection-dominated accretion flows, and we present a toy model which can explain the radio to sub-mm spectrum and polarization of $\operatorname{Sgr} \mathrm{A}^{*}$.
\end{abstract}

\section{INTRODUCTION}

Recent proper motion measurements of stars near the Galactic center have shown there to be a central mass of $3 \times 10^{6} M_{\odot}$ located within 0.03 arcseconds of the central radio source, Sgr $\mathrm{A}^{*}$, with an inferred mass density of $\sim 10^{13} M_{\odot} / p c^{3}$ (Genzel et al. 1998, Ghez et al. 1998, Ghez et al. 2000). The radio source is quite faint, with a bolometric luminosity of $\sim 10^{37} \mathrm{erg} / \mathrm{s}$, so that the mass to light ratio of the central object is $\sim 10^{-3} L_{\odot} / M_{\odot}$. The high mass density and low luminosity are strong evidence that a black hole lurks at the dynamical center. The accretion rate of matter by the black hole should be as large as $10^{-(4-5)} M_{\odot} / y r$ (Quataert, Narayan, \& Reid 1999; Coker \& Melia 1999). This is rather surprising given the rather low bolometric luminosity of the point source associated with Sgr A*; the inferred accretion efficiency is as low as $2 \times 10^{-6}$. Low efficiency might be achieved if viscous heating pumps energy into protons, which cannot cool as efficiently as electrons and proceed to carry their thermal energy into the black hole, a so-called "Advection-Dominated Accretion Flow" (or ADAF, lchimaru 1977, Narayan \& Yi 1994; Narayan, Yi, \& Mahadevan 1995), or if the gas has low angular momentum, and accretes spherically (Melia 1992). Alternatively, the accretion rate may be overestimated, or most infalling gas may be driven mechanically outwards by energy released from the gas which does accrete, either in a jet, wind, or convectively or magnetically driven outflow (Falcke, Mannheim, \& Biermann 1993, Igumenshchev \& Abramowicz 1999, 2000; Stone, Pringle, \& Begelman 1999; Quataert \& Gruzinov 2000; Narayan, Igumenshchev, \& Abramowicz 2000; Igumenshchev, Abramowicz,

CP556, Explosive Phenomena in Astrophysical Compact Objects, edited by H.-Y. Chang, et al (C) 2001 American Institute of Physics 1-56396-987-4/01/\$18.00 
\& Narayan 2000, Stone \& Pringle 2000). The actual accretion rate onto the black hole may be as low as $10^{-9} M_{\odot} / y r$.

One possible way to distinguish low and high accretion rates of the matter near the black hole is to measure the polarization and self-absorption frequency of the radio emission which is thought to be due to synchrotron-emitting plasma (Bower et al, 1999b, Agol 2000, Quataert \& Gruzinov 2000). Higher accretion rates imply higher gas densities and magnetic field strengths, leading to more Faraday rotation which will depolarize the synchrotron radiation and cause a higher self-absorption frequency. There is no definitive detection of linear polarization or self-absorption frequency of Sgr $\mathrm{A}^{*}$, consistent with the idea of a high accretion rate. However, recent tantalizing polarization observations by Aitken et al. (2000, hereafter A00) with the SCUBA array reveal that near $1 \mathrm{~mm}$, the radio source may be linearly polarized, and that the polarization angle changes with wavelength by about $90^{\circ}$, possibly indicating that the self-absorption frequency occurs around $0.5 \mathrm{~mm}$. If these observations are confirmed with higher angular resolution to avoid confusion from polarized dust emission, then the polarization will be strong evidence against a high accretion rate. We discuss synchrotron polarization in section 2 , the limits on the accretion rate in section 3, a toy model which can explain the current observations (if taken at face value) in section 4 , and summarize in section 5 .

\section{SYNCHROTRON THEORY}

In the synchrotron limit $(\gamma \gg 1)$ for an isotropic electron velocity distribution, some analytic results have been derived, which we now summarize (Ginzburg \& Syrovatskii 1965 \& 1969: GS ). For a uniform slab of electrons with a powerlaw distribution, $d n_{e} / d \gamma \propto \gamma^{-\xi}$ (with $\gamma_{\min } \leq \gamma \ll \gamma_{\max }$ such that electrons with $\gamma_{\min }$ and $\gamma_{\max }$ do not contribute to the frequency of interest), we can relate the magnetic field strength and electron density in the slab to the fluid-frame brightness temperature and the spectral turnover due to self-absorption. For $\xi=2$ and a uniform field $B_{\perp}$ (projected into the sky plane) we find $B_{\perp} \sim 2 T_{11}^{-2} \nu_{12} \mathrm{G}$ and $\tau_{C} \sim 3 \times 10^{-2} T_{11}^{4} \nu_{12} \gamma_{\text {min }}^{-1}$, where $T_{11}$ is the brightness temperature in units of $10^{11} \mathrm{~K}$ at the self-absorption frequency $\nu_{t}=10^{12} \nu_{12} \mathrm{~Hz}$ and $\tau_{C}$ is the Compton scattering optical depth of the emission region. For $\nu<\nu_{t}$, the emission is self-absorbed so $F_{\nu} \propto \nu^{5 / 2}$, while above this frequency the emission is optically-thin and $F_{\nu} \propto$ $\nu^{(1-\xi) / 2} \exp \left(-\nu / \nu_{\max }\right)$ where $\nu_{\max }=3 B_{\perp} e \gamma_{\max }^{2} /\left(4 \pi m_{e} c\right)$.

In the optically-thin regime, the polarization plane is perpendicular to the magnetic field with polarization $\Pi=(\xi+1) /(\xi+7 / 3)$, up to $100 \%$ for $\xi \gg 1$. In the optically-thick regime, $\Pi=-3 /(6 \xi+13$ ) (for $\xi>1 / 3$ ); the radiation polarized perpendicular to the magnetic field is absorbed more strongly than the opposite polarization, causing the radiation polarized along the magnetic field to dominate, switching the polarization angle by $90^{\circ}$, which changes the sign of $\Pi$. Numerical calculations show that the optically-thick polarization peaks at $|\Pi|=20 \%$ for $\xi=1 / 3$, but remains large for $0<\xi<2$. 
To compute the polarization near the self-absorption frequency requires a knowl-

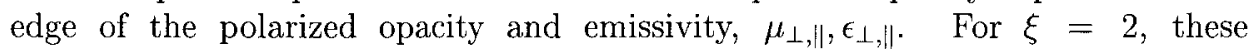
can be approximated as (GS): $\mu_{\perp, \|}=r_{s}^{-1}\left(\nu / \nu_{t}\right)^{-3}(1 \pm 3 / 4)$ and $\left(\epsilon_{\perp, \|} / \mu_{\perp, \|}\right)=$ $2 S_{t} / 9\left(\nu / \nu_{t}\right)^{5 / 2}(13 \pm 9) /(4 \pm 1)$ where $r_{s}$ is the size of the emission region, $\nu_{t}$ is the frequency for which the total source has an optical depth of unity (i.e. $\left.\tau=\mu r_{s}=1 / 2\left(\mu_{\perp}+\mu_{\|}\right) r_{s}=1\right), S_{t}$ is the source function near the frequency $\nu_{t}$, and the + or - signs go with the radiation emitted $\perp$ or $\|$ to the magnetic field, respectively. GS then express the polarization and emission for a slab with uniform magnetic field strength and direction, constant density, and size $r_{s}: I_{\perp}=\left(\epsilon_{\perp} / \mu_{\perp}\right)\left(1-\exp \left(-\mu_{\perp} r_{s}\right)\right), \quad I_{\|}=\left(\epsilon_{\|} / \mu_{|l|}\right)\left(1-\exp \left(-\mu_{\|} r_{s}\right)\right)$, and $\Pi=\left(I_{\perp}-I_{\|}\right) /\left(I_{\perp}+I_{\|}\right)$, where $I_{\perp}, I_{\|}$are the intensities $\left(\mathrm{erg} / \mathrm{cm}^{2} / \mathrm{s} / \mathrm{Hz} / \mathrm{sr}\right)$ with polarization perpendicular and parallel to the projected direction of the magnetic field on the sky.

For electron distributions which are highly peaked at a single energy (such as mono-energetic or relativistic Maxwellian) the polarization for $\nu \lesssim \nu_{t}$ is zero.

The Faraday effect rotates the polarization vector of photons emerging from different optical depths by different amounts, causing a cancellation in polarization (Agol \& Blaes 1996). The differential Faraday rotation angle within the source scales as $\Delta \theta=3.6 \times 10^{28} \tau_{\text {phot }} B \nu^{-2} \gamma_{\min }^{-2}$ (Jones \& O'Dell 1977), where $\tau_{p h o t}$ is the Compton optical depth of the photosphere. When optically thin, $\tau_{p h o t} \sim \tau_{C}$ is constant, so rotation is largest at the self-absorbed wavelength. When self-absorbed, $\tau_{\text {phot }}$ of the photosphere scales as $\nu^{\xi / 2+2}$, so the differential Faraday rotation angle $\propto \nu^{\xi / 2}$ (for $\xi>1 / 3$ ), again largest at the self-absorption wavelength. The differential rotation at $\nu_{t}$ is $\Delta \theta \sim 2 \pi g(\xi)\left(\theta_{b} / \gamma_{\min }\right)^{\xi} / \gamma_{\min }$, where $\gamma_{\min }$ is the minimum electron Doppler factor, $g(\xi)$ is a dimensionless factor of order unity, and $\theta_{b}$ is the brightness temperature in units of $m_{e} c^{2} / k_{B}$.

\section{LIMITS ON THE ACCRETION RATE}

The observations of polarization in Sgr $A^{*}$ provide the following constraints on emission models:

1) The differential Faraday rotation angle near $\nu_{t}$ must be $\ll \pi$.

2) The electron distribution must be non-thermal since the polarization due to a thermal electron distribution is suppressed when self-absorbed by a factor of $\exp (-\tau)$. If the beam correction by A00 is correct, then $\Pi \sim 12 \%$ at self-absorbed wavelengths, requiring $\xi \lesssim 2$.

3) The self-absorption frequency must lie near the change in polarization angle.

4) The component at lower frequencies must have no linear polarization.

5) The magnetic field must be ordered to prevent cancellation of polarization.

These constraints rule out several models proposed in the literature, as will be discussed in turn.

The low efficiency of an ADAF implies a higher accretion rate and thus higher density than for a high efficiency flow of the same luminosity and geometrical thick- 
ness. For $\operatorname{Sgr} \mathrm{A}^{*}$, an accretion rate of $\sim 10^{-(4-5)} M_{\odot} / \mathrm{yr}$ is inferred due to capture of gas in the vicinity of the black hole (Quataert, Narayan, \& Reid 1999; Coker \& Melia 1999), which is the value assumed in ADAF models. Assuming that the gas falls in at near the free-fall speed, one infers an electron density $n_{e}=10^{10}$ $\mathrm{cm}^{-3} \dot{m}_{-5} x^{-3 / 2}$ and a magnetic field strength of $B=10^{3} \mathrm{G}_{-5}^{1 / 2} x^{-5 / 4}\left(v_{A} / 0.1 v_{f f}\right)$, where $x$ is the radius of the emission region in units of $r_{g}=G M / c^{2}, \dot{m}_{-5}$ is the accretion rate in units of $10^{-5} M_{\odot} / \mathrm{yr}$, and $v_{A} / v_{f f}$ is the ratio of the Alfvén speed to the free-fall speed. These values imply a total Faraday rotation angle at the self-absorption frequency $\nu_{t}$ of $\Delta \theta \sim 10^{4} \dot{m}_{-5}^{3 / 2} \nu_{12}^{-2}\left(v_{A} / 0.1 v_{f f}\right)$. This value is so large that rotation of the emitted radiation leads to zero net polarization, so ADAFs are in direct conflict with the observed polarization. Only significant modifications of the model, such as a reduction in the accretion rate by a factor of $10^{-3}$, can reduce the Faraday rotation angle $\ll \pi$. An accretion rate of $10^{-8} M_{\odot} /$ yr is consistent with the observed luminosity if the accretion flow has a higher efficiency $\sim 2 \%$, no longer "advection-dominated." In addition, ADAF models assume a Maxwellian electron distribution, which cannot produce the observed switch in polarization angle. Finally, ADAFs predict a higher self-absorption frequency: Özel et al. (2000) find that $\nu_{t} \sim 5 \times 10^{12} \dot{m}_{-5}^{5 / 9} \mathrm{~Hz}$, which implies $\dot{M} \sim 4 \times 10^{-7} M_{\odot} /$ yr to be consistent with the observed $\nu_{t} \sim 5 \times 10^{11} \mathrm{~Hz}$. The accretion rate might be reduced if there is significant gas lost by a wind or jet (Begelman \& Blandford 1999; Quataert \& Narayan 1999) or if the Bondi rate is reduced by heating the infalling gas with heat carried outwards by a convection-dominated accretion flow, or "CDAF" (Igumenshchev \& Abramowicz 1999, 2000; Stone, Pringle, \& Begelman 1999; Quataert \& Gruzinov 2000; Narayan, Igumenshchev, \& Abramowicz 2000; Igumenshchev, Abramowicz, \& Narayan 2000).

The model of Melia (1992) is rather similar to the ADAF model, and thus suffers the same problems: the high accretion rate implies high density which is inconsistent with the observed polarization.

Beckert \& Duschl (1997) considered several 1-zone, quasi-monoenergetic and thermal emission models for the synchrotron emission. These electron distributions do not produce a swing in polarization angle by 90 degrees since the polarization is suppressed when self-absorbed. Their model does produce a self-absorption frequency near the correct frequency, however. Falcke, Mannheim, \& Biermann (1993) present a disk-plus-jet model which assumes a tangled magnetic field topology which would erase any polarization. However, an ordered magnetic field would be a small change to their model which might bring it into line with the polarization observations.

\section{TOY MODEL}

Now, we attempt to construct a model consistent with all of the data, using uniform emission regions for simplicity. Typical optically-thin AGN spectra show $\xi \sim 2-3$; since $\xi=2$ is consistent with the polarization from $A 00$, we fix $\xi=2$ in 
our model fits. The model parameters for the polarized component are $S_{t}=6 \mathrm{Jy}$, $\nu_{t}=550 \mathrm{GHz}$ (corresponding to $\lambda=0.55 \mathrm{~mm}$ ), and $\nu_{\max } \sim 5000 \mathrm{GHz}$ (Figure 1).

To explain the lack of polarization and spectral slope flatter than $5 / 2$, we require an additional component which is unpolarized and has a cutoff near $1 \mathrm{~mm}$ so that it doesn't dilute the polarization at shorter wavelengths. Since Sgr A* has a spectral slope of $1 / 3$ at $\mathrm{mm}$ wavelengths and appears to have a spectral turnover at $1 \mathrm{GHz}$, we model the spectrum as a monoenergetic electron distribution with energy $\gamma$ and zero polarization (due to Faraday depolarization or tangled magnetic field) which becomes self-absorbed at low frequency (Beckert \& Duschl 1997). For the unpolarized component, we find $F_{\nu}=1.3\left(\nu / \nu_{\max }\right)^{1 / 3} \exp \left(-\nu / \nu_{\max }\right)$ Jy with $\nu_{\max } \sim$ $50 \mathrm{GHz}$, and $\nu_{t} \sim 1 \mathrm{GHz}$ (Figure 1).

Figure 1 compares the model to the data. To compare the polarization, we have plotted the Stokes' parameter that lies at $83^{\circ}$. Remarkably, the polarization should rise to $\sim 100 \%$ at even shorter wavelengths.

\section{DISCUSSION}

Krichbaum et al. (1998) report a source radius of $55 \mu$ as at $1.4 \mathrm{~mm}$ from VLBI observations; this corresponds to $19 r_{g}$. The source size may be smaller at higher frequencies, but we expect the radius of the emission region to be greater than the size of the event horizon of the black hole, which has an apparent size of $\sim$ $5 r_{g} \sim 15 \mu$ as projected on the sky (including gravitational bending, Bardeen 1973), so we use an intermediate size in further estimates. The flux of the fitted model at the self-absorption frequency, $\nu_{t}=550 \mathrm{GHz}$, is $\sim 9 \mathrm{Jy}$. This implies a brightness temperature in the emission frame $T_{b} \sim 1.6 \times 10^{10}\left(r_{s} / 10 r_{g}\right)^{-2} \Gamma^{-1} \mathrm{~K}$, where $r_{s}$ is the size of the source (we have assumed the area of the source is $\pi r_{s}^{2}$ ) and $\Gamma$ is the bulk Doppler boost parameter. For a steeply falling electron number distribution, $k T_{b} \sim$ $4 \gamma m_{e} c^{2}$ (for $\xi=2$ ), where $\gamma m_{e} c^{2}$ is the energy of the emitting electrons, implying $\gamma \sim 10\left(r_{s} / 10 r_{g}\right)^{-2} \Gamma^{-1}$ for the electrons at the self-absorption frequency. Using the formulae from $\S 2$, we find: $B_{\perp}=350\left(r_{s} / 10 r_{g}\right)^{4} \Gamma G, \tau_{C}=10^{-5}\left(r_{s} / 10 r_{g}\right)^{-8} \Gamma^{-5}$, and $\gamma_{\max }=50\left(r_{s} / 10 r_{g}\right)^{-2} \Gamma^{-1}$, implying $n_{e} \sim 6 \times 10^{6}\left(r_{s} / 10 r_{g}\right)^{-9} \Gamma^{-5} \mathrm{~cm}^{-3}$. The ratio of magnetic to rest-mass energy density is $B^{2} /\left(8 \pi n_{e} m_{p} c^{2}\right) \sim 1\left(r_{s} / 10 r_{g}\right)^{17} \Gamma^{9 / 2}$ for an electron-proton plasma, indicating a relativistic Alfvén speed. The Faraday rotation angle at $\nu_{t}=5.5 \times 10^{11} \mathrm{~Hz}$ is $\Delta \theta \sim 350\left(r_{s} / 10 r_{g}\right)^{-4} \Gamma^{-2} \gamma_{\text {min }}^{-3}$, assuming $B_{\|} \sim B_{\perp}$. For $r_{s} \sim 10 r_{g}, \gamma_{\min }$ can be as large as 4, reducing $\Delta \theta$ to 5 ; for $r_{s} \sim 5 r_{g}$, $\gamma_{\min }$ can be as large as 20 reducing $\Delta \theta$ to $\sim 0.6$. Alternatively, if the synchrotron emission is due to a pair plasma, Faraday rotation will be reduced by the ratio of the proton number density to the pair number density. The rotation angle is further reduced at the observed wavelengths by a factor $\sim \nu / \nu_{t}$. The high energy cutoff for the electron distribution may be due to synchrotron cooling since $t_{\text {cool }}=8 \times 10^{8} \gamma_{\max }^{-1} B^{-2} \sim 6\left(r_{s} / 10 r_{g}\right)^{-6} \Gamma^{-3} \mathrm{sec}$, similar to the dynamical time, $t_{D} \sim 13 x^{-3 / 2}$ sec. Given the strong scaling of quantities with the unknown $r_{s}$ and $\Gamma$, the above estimates can only be improved with future observations. 
The unpolarized emission component dominates at $\sim 7 \mathrm{~mm}$, where Lo et al. (1998) measure a source size of $\sim 5 \times 10^{13} \mathrm{~cm}$. The self-absorption frequency then requires $\gamma \sim 400, B \sim 0.1 \mathrm{G}$, and $n_{e} \sim 4 \times 10^{5} \mathrm{~cm}^{-3}$. Though somewhat ad-

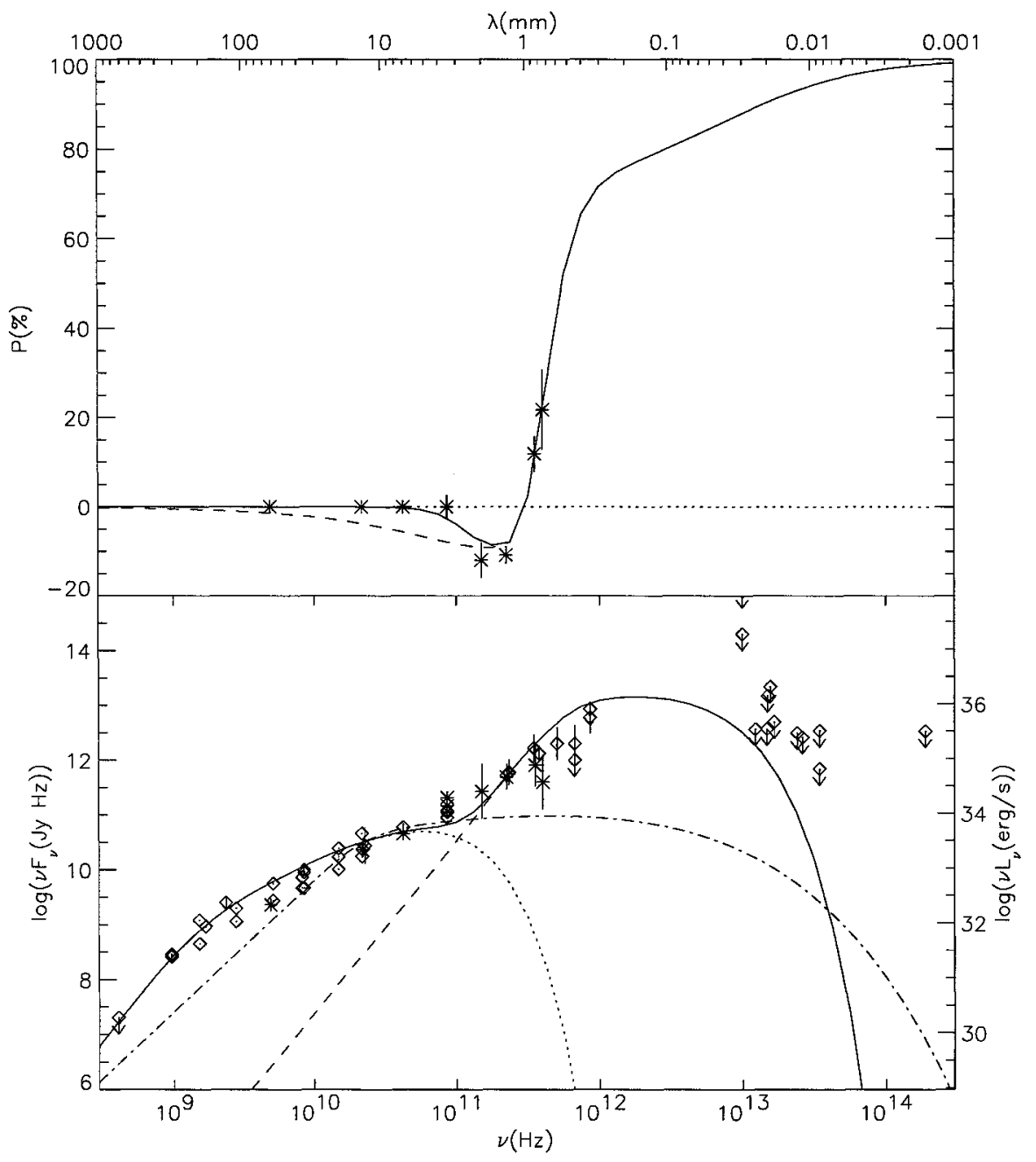

FIGURE 1. Fig. 1: Polarization and spectral energy distribution of $\mathrm{Sgr} \mathrm{A}^{*}$ compared to model. The dashed line shows the polarized component, the dotted line the unpolarized, mono-energetic component, and the solid line the sum of the two. The dot-dash line shows the maximum CDAF model (assumed to be unpolarized; the total polarization is similar if the CDAF replaces the monoenergetic component). The diamonds are the data compiled by Narayan et al. (1995), while the asterisks are the data from Bower et al. (1999a,b) and A00. 
hoc, this model reproduces the spectrum well. The Faraday rotation parameter is rather small, so depolarization requires field which is tangled on a scale $\sim 100$ times smaller than the size of the emission region.

Since the self-absorption frequency occurs at $\sim 500 \mu \mathrm{m}$, it will be possible to image shadow of a black hole from the ground using VLBI, providing a direct confirmation of the existence of an event horizon (Falcke, Melia, \& Agol 2000). Future sub-mm polarimetric VLBI observations might show rotation of the polarization angle near the black hole, a general relativistic effect which becomes stronger for a spinning black hole (Connors, Stark, \& Piran 1980).

This contribution is an abridged version of Agol (2000).

\section{REFERENCES}

1. Agol, E., 2000, ApJL, 538, 121

2. Aitken, D. K., et al., 2000, ApJ, in-press, astro-ph/0003379, A00

3. Begelman, M. C. \& Blandford, R. D., 1999, MNRAS, 303, L1

4. Bower, G. C., Backer, D. C., Zhao, J. H., Goss, M., \& Falcke, H. 1999a, ApJ, 521, 582

5. Bower, G. C., Wright, M. C. H., Backer, D. C., \& Falcke, H. 1999b, ApJ, 527, 851

6. Beckert, T. \& Duschl, W. J., 1997, A\& A, 328, 95

7. Coker, R. \& Melia, F., 1999, ApJ, 511, 750

8. Falcke, H., Mannheim, K., \& Biermann, P. L., 1993, A\&A, 278, L1

9. Falcke, H., Melia, F., \& Agol, E., 2000, ApJ, 528, L13

10. Genzel, R., Eckart, A., Ott, T., \& Eisenhauer, F., 1997, MNRAS, 291, 219

11. Ghez, A. M., Klein, B. L., Morris, M., \& Becklin, E. E. 1998, ApJ, 509, 678

12. Ghez, A. M., Morris, M., Becklin, E. E., Tanner, A., \& Kremenek, T., 2000, Nature, Sept. 21 issue, astro-ph/0009339

13. Ginzburg, V. L. \& Syrovatskii, S. I., 1965, ARA\&A, 3, 297, GS

14. Ginzburg, V. L. \& Syrovatskii, S. I., 1969, ARA\&A, 7, 375, GS

15. Ichimaru, S., 1977, ApJ, 214, 840

16. Igumenshchev, I. V. \& Abramowicz, M. A., 1999, MNRAS, 303, 309

17. Igumenshchev, I. V. \& Abramowicz, M. A., 2000, astro-ph/003397

18. Krichbaum, T. P. et al. 1998, A\&A, 335, L106

19. Lo, K. Y., Shen, Z. Q., Zhao, J. H., \& Ho, P. T. P. 1998, ApJ, 508, L61

20. Melia, F., 1992, ApJ, 387, L25

21. Narayan, R. \& Yi, I., 1994, ApJ, 428, L13

22. Narayan, R., Yi, I., \& Mahadevan, R., 1995, Nature, 374, 623

23. Narayan, R., Igumenshchev, I. V. \& Abramowicz, M. A., 2000, astro-ph/9912449

24. Özel, F., Psaltis, D., \& Narayan, R., 2000, ApJ, in press, astro-ph/0004195

25. Quataert, E., Narayan, R., \& Reid, M. J., 1999, ApJ, 517, L101

26. Quataert, E. \& Gruzinov, A., 2000, ApJ, in press, astro-ph/9912440

27. Stone J. M., Pringle J. E., \& Begelman M. C., 1999 MNRAS, 310, 100

28. Stone J. M. \& Pringle J. E., 2000, astro-ph/0009233 